\title{
Conservación ex situ de Puya loca Madriñan (Bromeliaceae) y registro de una segunda localidad en los páramos de Colombia
}

\author{
Adriana Pico-V \\ Subdirección Científica, Jardín Botánico de Bogotá “José Celestino Mutis”, Bogotá, Colombia
}

\begin{abstract}
Resumen
Puya loca Madriñan fue descrita en el año 2015 y hasta ahora se ha encontrado en dos localidades de los páramos de la cordillera Oriental de Colombia: la primera, en el complejo de páramos de Chingaza (localidad del tipo), al nororiente de Bogotá, y la segunda corresponde a un nuevo registro en el municipio de Chipaque, al suroriente de Bogotá. Esta especie se desarrolla sobre suelos bien drenados, en grietas de roca con poco sustrato o sobre plantas no vasculares, en plena exposición al sol y el viento. Con la finalidad de conservar la variabilidad genética de la especie en condiciones ex situ y, posteriormente, in situ, se evaluaron las condiciones naturales del hábitat de la localidad en Chipaque y se desarrollaron experimentos de propagación ex situ en el Jardín Botánico de Bogotá (JBB). Asimismo, se estudió la viabilidad mediante los porcentajes de germinación y durante 1,3 años los rasgos de crecimiento y desarrollo de las plántulas. En $P$. loca la germinación está regulada por la luz (especie fotoblástica positiva), y las semillas, con 14 meses de conservación en condiciones naturales, germinaron ex situ entre los 19 y 30 días de la siembra (75\% de germinación). Las plántulas de 35 días desarrollaron una o dos hojas y a los 61 días presentaban entre dos y tres hojas. Con 15 meses de edad, las plántulas sembradas en $6 \mathrm{~cm}$ de turba desarrollaron cinco a siete hojas y alcanzaron 6 a $7 \mathrm{~cm}$ de diámetro, en tanto que las plántulas sembradas en 2,5 a $3 \mathrm{~cm}$ de turba desarrollaron cuatro a seis hojas y alcanzaron 3 a $4 \mathrm{~cm}$ de diámetro, con un crecimiento de menor vigor. Actualmente se conservan 100 plántulas para el estudio ex situ del ciclo de la especie, que, eventualmente, se reintroducirán en su hábitat natural. (C) 2016. Acad. Colomb. Cienc. Ex. Fis. Nat.
\end{abstract}

Palabras clave: Puya; Bromelias; Cardón; Páramo; Propagación sexual; Colombia.

Ex situ conservation of Puya loca Madriñan (Bromeliaceae), and second report in Colombia

\begin{abstract}
Puya loca Madriñan has been found in two locations in the páramos of the Eastern Andes Cordillera in Colombia. The first location is the Chingaza páramo complex (the type location), northeast from Bogotá, and the second is a new location for the species in the municipality of Chipaque, southeast from Bogota. This species grows in welldrained soils, in rock cracks with little substrate or in non-vascular plants, and fully exposed to sun and wind. In this study, the natural habitat conditions were evaluated in the second location and then ex situ experiments were carried out in the Jardín Botánico de Bogotá (JBB). The seeds viability was established using germination rates, growth traits, and seedlings development in a period of 1.3 years. The germination in this species was regulated by light (positive photoblastic), and the seeds conserved for 14 months under natural conditions germinated in ex situ conditions between 19 and 30 days after planted (75\% germination rate). The 35 days-old seedlings formed 1 or 2 leaves and after 61 days the same seedlings formed 2 to 3 leaves. After 15 months, seedlings planted in $6 \mathrm{~cm}$ of peat developed between 5 to 7 leaves and reached a diameter of 6 to $7 \mathrm{~cm}$, while seedlings planted in 2,5 to $3 \mathrm{~cm}$ of peat got 4 to 6 leaves and 3 to $4 \mathrm{~cm}$ of diameter, and grew with less vigor. At present, we are conserving 100 seedlings to study the ex situ cycle for this species. Eventually these individuals will be reintroduced in their natural habitat. (C) 2016. Acad. Colomb. Cienc. Ex. Fis. Nat.
\end{abstract}

Key words: Puya; Bromeliads; Cardón; Páramo; Sexual propagation; Colombia.

\section{Introducción}

En Colombia, la familia Bromeliaceae está conformada por 543 especies, de las cuales $204(37,6 \%)$ son endémicas (Betancur, 2016), 153 (28,2 \%) están bajo algún nivel de amenaza y 25 de estas últimas pertenecen al género Puya (Betancur \& García, 2006; Ministerio de Ambiente y Desarrollo Sostenible, 2014). Los estudios sobre la germinación, el crecimiento y el desarrollo, los tipos y el efecto de la conservación de las semillas, la posible dormancia y el efecto del fitocromo de las bromelias en

\footnotetext{
Correspondencia:

Adriana Pico-V, apico@jbb.gov.co, adri.pico.villalobos@gmail.com

Recibido: 09 de mayo de 2016
}

Aceptado: 08 de noviembre de 2016 
Colombia y el Neotrópico son escasos, tal como puede constatarse en la Tabla 1 (Pico, 2002, 2014a, 2014b, 2014c y 2015; Vadillo, et al., 2004; Mora, et al., 2007).

Puya loca Madriñan fue descrita recientemente para Colombia, en el municipio de La Calera, departamento de Cundinamarca, cerca del límite nororiental de Bogotá (The Plant List, 2013; Madrinán, 2015; The International Plant Names Index, 2016). La única población que se conocía en el momento de la descripción se encontraba en el complejo de páramos de Chingaza, en el cerro Tunjaque, reserva Tierraleja, a $3.550 \mathrm{msnm}$, donde "forma grupos o masas densas de rosetas en acantilados rocosos y [...] se desarrolla en pequeños parches aislados de páramos" (Madriñán, 2015). Sin embargo, en el presente trabajo se registró otra población de esta especie en el municipio de Chipaque, con lo cual se amplía su distribución.

En este estudio se presentan las alternativas de conservación de una nueva especie endémica de los páramos centrales de la cordillera Oriental muy amenazada por el cambio del uso de la tierra en las áreas aledañas a Bogotá

Tabla 1. Resultados relevantes de investigaciones sobre germinación, crecimiento y desarrollo de Puya

\begin{tabular}{|c|c|c|c|c|}
\hline Especie & $\begin{array}{l}\text { Factores evaluados y } \\
\text { lugar de las observaciones }\end{array}$ & $\begin{array}{l}\text { Tiempo de } \\
\text { germinación }\end{array}$ & Porcentajes de germinación & Investigación \\
\hline Puya bicolor Mez & $\begin{array}{l}\text { Ex situ, luz, semillas } \\
\text { no almacenadas, recién } \\
\text { recolectadas. Crecimiento } \\
\text { y desarrollo (número de. } \\
\text { hojas). En JBB, Colombia. } \\
2.550 \mathrm{msnm}\end{array}$ & Día 21 & $\begin{array}{l}\text { Día } 21 \text { ( } 28 \% \text { a } 55 \% \text { por réplica), } \\
\text { especialmente hasta el día } 28 \text { (máximo } \\
98 \% \text { por réplica). Finalizó en } 5 / 6 \text { réplicas } \\
\text { el día } 42(94 \%) \text {. Hasta el día } 129 \text { en una } \\
\text { réplica }(80 \%) \text {. Para el día } 161 \text { habían } \\
\text { desarrollado hasta } 10 \text { hojas. }\end{array}$ & Pico, 214a \\
\hline Puya santosii Cuatrec. & $\begin{array}{l}\text { Ex situ, luz, semillas } \\
\text { no almacenadas, recién } \\
\text { recolectadas. Crecimiento } \\
\text { y desarrollo (número de } \\
\text { hojas). En JBB, Colombia. } \\
2.550 \mathrm{msnm}\end{array}$ & Día 28 hasta día 87 & $\begin{array}{l}\text { Mínimo } 21 \% \text { en el día inicial (día } 28 \text { ) } \\
\text { hasta } 48 \% \text { en el día } 87 . \text { Las plántulas } \\
\text { habían desarrollado hasta siete hojas } \\
\text { en el día } 155 .\end{array}$ & Pico, 2014 \\
\hline $\begin{array}{l}\text { Puya cryptantha } \text { Cuatrec. } \\
\text { y Puya trianae Baker }\end{array}$ & $\begin{array}{l}\text { Semillas enterradas in } \\
\text { situ hasta } 12 \text { meses y en } \\
\text { condiciones de laboratorio } \\
\text { con luz y dos poblaciones } \\
\text { (ex situ). En Bogotá, } \\
\text { Colombia }\end{array}$ & $\begin{array}{l}\text { Mes } 5 \text { (día } 150 \text { como } \\
\text { mínimo) en tres de } \\
\text { cuatro poblaciones }\end{array}$ & $\begin{array}{l}\text { Diferencias en la viabilidad por los } \\
\text { factores evaluados. Mayor germinación } \\
\text { en condiciones de laboratorio. } \\
\text { La viabilidad en condiciones de } \\
\text { laboratorio se reportó como alta durante } \\
12 \text { meses, con proporción de semillas } \\
\text { viables de } 90 \% \text {, y valores superiores } \\
\text { a } 85 \% \text { en la mayoría de los meses, y } \\
\text { germinación en condiciones de } \\
\text { laboratorio superiores a } 90 \% \text { cuando } \\
\text { se las hidrataba y se mantenían bajo } \\
\text { luz natural }\end{array}$ & Mora, et al., 2007 \\
\hline Puya berteroniana Mez & Temperatura & Sin datos & $\begin{array}{l}\text { Mayor germinación a } 15^{\circ} \mathrm{C} \text { y } \\
\text { disminución al exponerlas a } 25^{\circ} \mathrm{C}\end{array}$ & $\begin{array}{l}\text { Smith \& Downs } \\
(1974)\end{array}$ \\
\hline Puya raimondii Harms & $\begin{array}{l}\text { Germinación y viabilidad } \\
\text { en el tiempo }\end{array}$ & $\begin{array}{l}90 \% \text { de germinación } \\
\text { a los } 32 \text { días }\end{array}$ & Mantuvo la viabilidad por seis meses & $\begin{array}{l}\text { Hartmann (1981), en } \\
\text { Vadillo, et al., } 2004\end{array}$ \\
\hline Puya raimondii Harms & $\begin{array}{l}\text { Germinación y viabilidad } \\
\text { en el tiempo }\end{array}$ & $\begin{array}{l}99 \% \text { de germinación a } \\
\text { los } 18 \text { días }\end{array}$ & Mantuvo la viabilidad por años & $\begin{array}{l}\text { Rivera (1985), en } \\
\text { Vadillo, et al., } 2004\end{array}$ \\
\hline Puya raimondii Harms & $\begin{array}{l}\text { Selección morfológica } \\
\text { por contenido de humedad, } \\
\text { temperatura y luz y } \\
\text { oscuridad }\end{array}$ & $\begin{array}{l}\text { Nueve días en todos } \\
\text { los tratamientos } \\
\text { (viabilidad de } \\
41,85 \% \text { ) }\end{array}$ & $\begin{array}{l}\text { Mayor germinación a } 11^{\circ} \mathrm{C}(35 \% \\
\text { a } 85 \%) \text { que a } 25^{\circ} \mathrm{C}(24 \% \text { a } 44 \%) \text {. } \\
\text { Mayor germinación con luz }(24 \%) \\
\text { que en la oscuridad (menos de } 6 \%) \text {. } \\
\text { Fotoblásticas positivas, y, por lo tanto, } \\
\text { controladas por el fitocromo }\end{array}$ & Vadillo, et al. 2004 \\
\hline Género Puya & Temperatura & (no dato) & $\begin{array}{l}\text { Correlación inversa en la germinación } \\
\text { del género }\end{array}$ & Smith \& Downs, 1974 \\
\hline $\begin{array}{l}\text { Algunas especies de } \\
\text { Puya y Espeletia }\end{array}$ & Luz y oscuridad & Sin datos & $\begin{array}{l}\text { Mayores porcentajes de germinación } \\
\text { en luz que en oscuridad }\end{array}$ & $\begin{array}{l}\text { Smith, 1974; } \\
\text { Guariguata \& Azócar, } \\
\text { 1988; Ochoa (1994) } \\
\text { en Mora, et al., } 2007\end{array}$ \\
\hline Pitcairnia flammea Lindl. & Temperatura & Sin datos & $\begin{array}{l}\text { Disminución de porcentaje de } \\
\text { germinación en } 17 \% \text { con aumento } \\
\text { en la temperatura de } 15 \text { a } 25^{\circ} \mathrm{C} \text {. }\end{array}$ & $\begin{array}{l}\text { Mercier \& Guerreiro } \\
\text { (1990) en Vadillo, } \\
\text { et al., } 2004\end{array}$ \\
\hline Muchas bromelias & Luz y oscuridad & Sin datos & $\begin{array}{l}\text { Las radiaciones cíclicas o luz continua } \\
\text { promovieron la germinación }\end{array}$ & Smith \& Downs, 1974 \\
\hline
\end{tabular}


debido a las dinámicas de expansión agrícola en los ecosistemas de subpáramo y páramo, de expansión del área urbanizable y de la extracción de arena de cantera (Secretaría Distrital de Ambiente, 2007; Guhl, 2015; Alcaldía Mayor de Bogotá, 2016).

Dada la relevancia de esta especie, el Jardín Botánico de Bogotá (JBB) cumple con sus objetivos de garantizar la conservación del patrimonio genético de la biodiversidad de plantas del bosque altoandino y del páramo, especialmente de plantas endémicas y amenazadas, así como de nuevas especies y de otras importantes de uso local (Jardín Botánico de Bogotá, 2015), mediante programas de investigación de conservación ex situ (Pico, 2002, 2014a, 2014b, 2014c y 2015; Jardín Botánico de Bogotá, 2015).

\section{Metodología}

En octubre de 2013, el personal del JBB recolectó semillas y plantas de P. loca (Pico, 2014c y 2015) durante salidas de campo al municipio de Chipaque, vereda Calderitas, páramo de Calderitas ( $4^{\circ} 24^{\prime} 22,1^{\prime \prime} \mathrm{N} ; 74^{\circ} 6^{\prime} 53,8^{\prime \prime}$ O), a 3.274 msnm, cerca de la localidad de Usme en Cundinamarca. Se registraron las condiciones del hábitat de las poblaciones de Chipaque y los primeros registros ex situ de germinación, crecimiento y desarrollo de $P$. loca hasta la edad de 1,3 años.

Las semillas recolectadas se almacenaron durante 14 meses en las condiciones ambientales de Bogotá (2.550 $\mathrm{msnm}$ ); la hipótesis inicial planteaba que se registrarían tasas de germinación bajas debido al tiempo transcurrido y el tipo de almacenamiento, pues en otras especies de Puya se ha reportado una reducción de la viabilidad con el tiempo y una correlación inversa con la temperatura en que se almacenan las semillas, como lo han señalado para el género Puya, en general, Smith \& Downs (1974), para Puya berteroniana Mez, también Smith \& Downs (1974), para Puya raimondii, Hartmann, 1981 (en Vadillo, et al., 2004) y para otra bromelia, Pitcairnia flammea Lindl, Mercier \& Guerreiro, 1990 (en Vadillo, et al., 2004). Otros investigadores sostienen que para la especie más grande del mundo (Puya raimondii), la viabilidad de la germinación se mantiene durante años (Rivera, 1985, en Vadillo, et al., 2004) (Tabla 1).

En todas las especies de Puya relacionadas en la Tabla 1 también se ha registrado una mayor viabilidad por conservación a bajas temperaturas comparadas con la temperatura ambiente; lo mismo se ha reportado para el género Puya, especialmente P. berteroniana Mez (Smith \& Downs, 1974), P. raimondii (Vadillo, et al., 2004) y $P$. flammea Lindl. (Mercier \& Guerreiro, 1990, en Vadillo, et al., 2004).

Desde 1974 se viene reportando en diversas investigaciones una respuesta positiva de germinación de las semillas de muchas especies de bromelias en condiciones de luz (Smith \& Downs, 1974), lo cual las determina como especies fotoblásticas positivas, característica también reportada para algunas especies de Puya (Smith, 1974; Guariguata \&
Azócar, 1988; Ochoa, 1994, en Mora, et al., 2007) y para las especies P. raimondii (Vadillo, et al., 2004), Puya cryptantha Cuatrec. y Puya trianae Baker (Mora, et al., 2007), así como para Puya bicolor Mez (Pico, 2014a) y Puya santosii Cuatrec. (Pico, 2014b).

Area de estudio. La fase de campo se hizo en el municipio de Chipaque (Pico, 2014c y 2015). Se herborizó un espécimen para el herbario del JBB y se recolectaron siete individuos vivos y dos infrutescencias maduras, de las cuales se obtuvieron 600 semillas (Figuras 1 a 3). Se generaron condiciones ex situ en la zona de páramo del JBB similares a las de la población visitada, se sembraron las plantas de manera gregaria en la colección viva y se codificaron como accesiones 24598 a 24604 (Pico, 2014c y 2015).

Germinación, crecimiento y desarrollo. Germinación. Se investigó la viabilidad de las semillas de P. loca con 14 meses de conservación en las condiciones ambientales del JBB. Las dos infrutescencias se secaron en bandeja destapada en un laboratorio durante 15 días y, posteriormente, las semillas se guardaron en bolsas plásticas con cierre hermético.

En un invernadero del JBB se sembraron 40 semillas en una bandeja plástica de $30 \times 15 \times 3 \mathrm{~cm}$ de profundidad, con pequeñas perforaciones en el fondo, tapa plástica transparente para mantener la humedad relativa, en turba comercial humedecida previamente y en condiciones de luz natural; se regaron dos veces por semana a capacidad de campo. Debido a las festividades de fin de año del 2014, se intentó hacer registros de germinación frecuentes, aunque no de forma estandarizada (en los días 5, 19, 21, 29, 35, $43,50,57,61$ y 66 después de la siembra). Además, se sembraron 460 semillas no experimentales en condiciones similares con destino al stock adicional de plántulas para conservación de la especie.

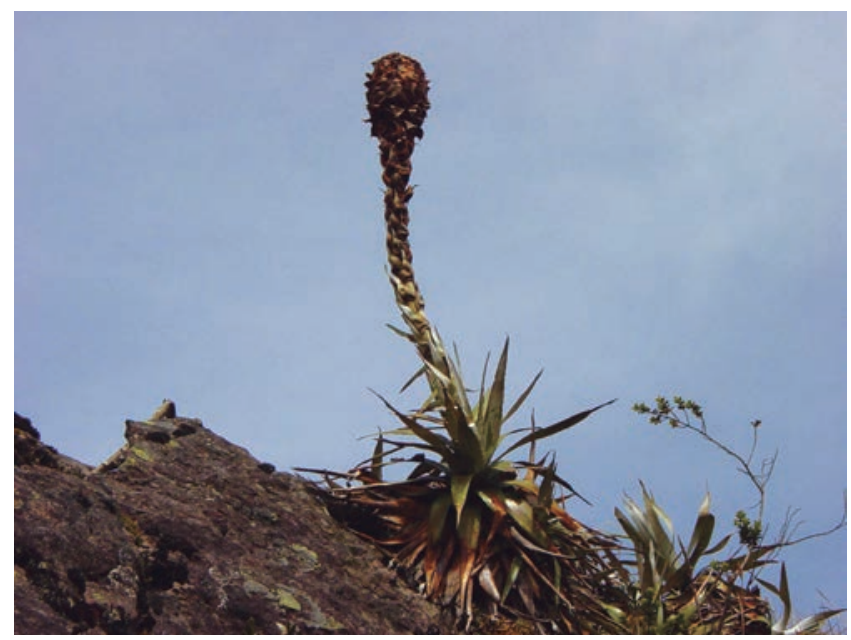

Figura 1. Planta madura de Puya loca Madriñan sobre grietas de roca. Páramo de Calderitas, municipio de Chipaque, Cundinamarca, 2013. Fotografía: Vilma Jaimes S. 




Figura 2. Inflorescencia de Puya loca en la matriz de páramo. Al fondo cultivos. Páramo de Calderitas, 2016.

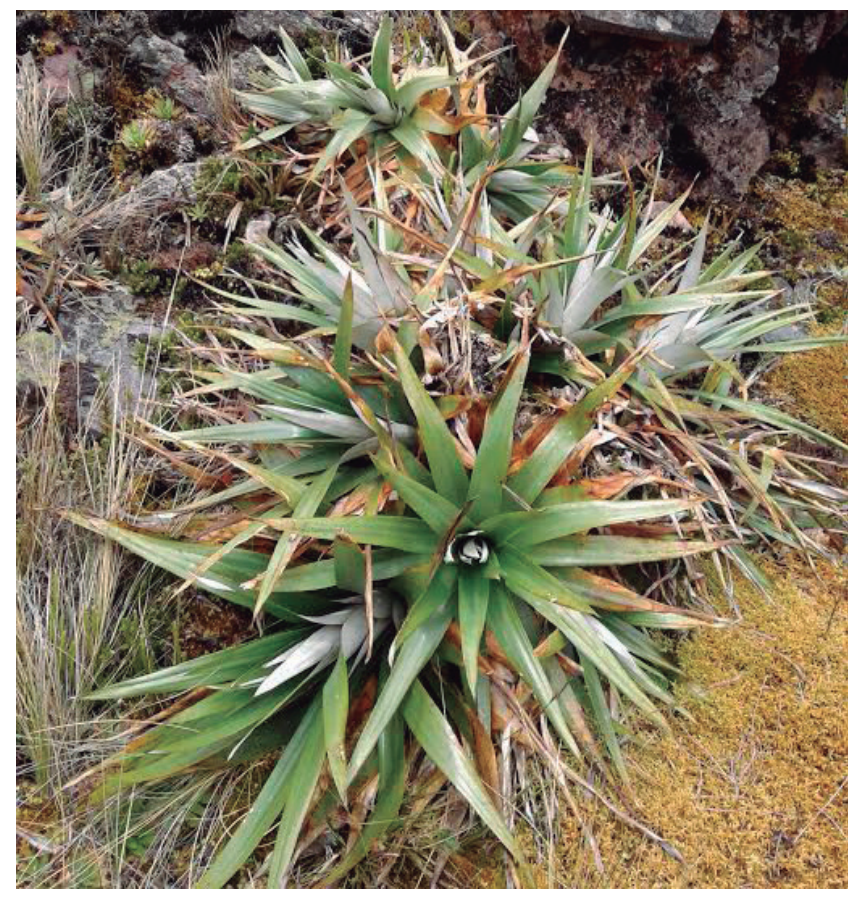

Figura 3. Agregación de hasta 10 individuos sobre grietas de rocas. Páramo de Calderitas, 2016.
Crecimiento y desarrollo. El número de hojas y la altura de las plántulas se registraron en los días 19, 29, 35 y 61 después de la siembra. Posteriormente, para comparar de manera global el crecimiento y desarrollo de la nueva especie en dos momentos, 9,5 y 13 meses después de la siembra (días 287 y 448, respectivamente), parte de las 30 plántulas experimentales y cientos de plántulas no experimentales se mantuvieron en las bandejas de germinación (sustrato de 2,5 a $3 \mathrm{~cm}$ de profundidad) y otras se resembraron en cuatro materas de $25 \mathrm{~cm}$ de diámetro con turba de $6 \mathrm{~cm}$ de profundidad.

\section{Resultados y discusión}

Los individuos de $P$. loca recolectados en Chipaque presentaban las rosetas densas de 1 a 1,2 $\mathrm{m}$ de altura, hojas de 0,9 a $1,1 \mathrm{~m}$ de longitud, gruesas, parcialmente deflexas, con el haz verde claro, el envés gris a verde muy claro, la inflorescencia recta, el escapo amarillo claro a verde claro, folioso, las brácteas deflexas, la cabezuela densa, con brácteas café oscuro, las flores morado oscuro muy evidentes y emergentes, y con senescencia en color vino tinto a morado muy oscuro (Madriñán, 2015) (Figuras 1 a 3). Las plantas de Chipaque crecen plenamente expuestas al sol, en terrenos con pendiente de $0^{\circ}$ a $45^{\circ}$ y en suelos drenados o sobre hundimientos o grietas en rocas, de forma solitaria o en grupos de hasta cinco individuos usualmente, y pocas veces en agregaciones de hasta diez individuos pequeños vegetativos.

En el lugar se registró el desarrollo de $P$. loca sobre suelos secos, bien drenados y sobre grietas en rocas, donde previamente se han desarrollado pequeñas capas de sustrato o tierra con plantas no vasculares (musgos, líquenes y helechos), es decir que las condiciones in situ de desarrollo de esta especie son similares en ambas poblaciones de plantas.

Puya loca se ha encontrado en dos páramos de la cordillera Oriental de Colombia, en los municipios de Chipaque y La Calera, por lo que es muy probable que se encuentre en otras áreas de páramo entre esas dos ubicaciones, como las localidades de Usme, Santafé y Usaquén en Bogotá, y en Cundinamarca en los municipios de Une, Ubaque y Choachí.

Germinación. La germinación de P. loca se inició 19 días después de la siembra en ocho plántulas ( $20 \%)$, en el día 21 se registraron 17 plántulas $(42,5 \%)$ y entre los días 29 y 61 después de la siembra, 30 plántulas (75\%) (Figuras 4A y 4B). Las semillas de P. loca son fotoblásticas positivas, es decir, germinan en condiciones de luz, hecho que también ocurre en las especies de Puya mencionadas en la Tabla 1. Por otra parte, el porcentaje de germinación se considera alto, dada la correlación entre la reducción de la viabilidad por el tipo y el tiempo de conservación de semillas en esta especie y la de las especies de Puya señaladas en la Tabla 1. Esta prolongada capacidad de reproducción de $P$. loca permitiría establecer poblaciones con semillas liberadas un año antes en condiciones in situ y ex situ, hecho que pocas veces se registra en especies de Puya (observaciones personales de 

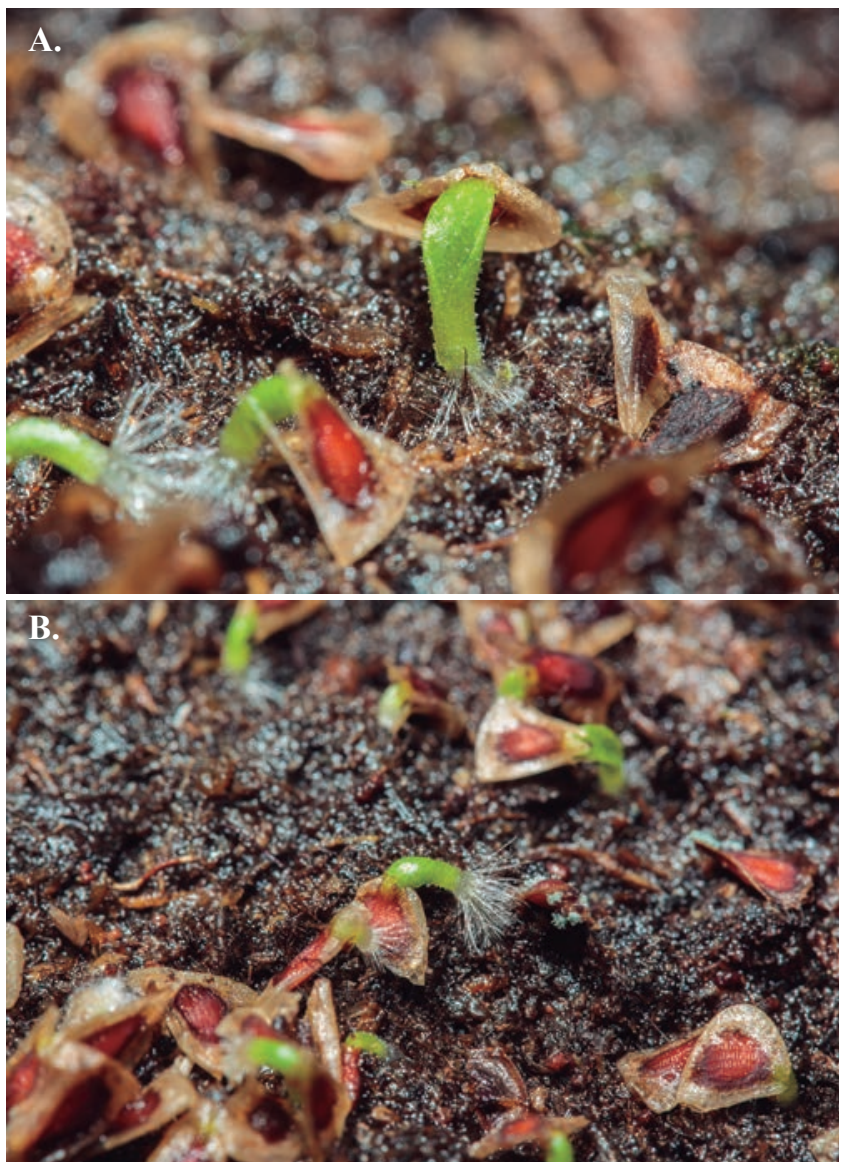

Figura 4. Plántulas con una hoja y semillas sin germinar 21 días después de la siembra, 2015. A. Plántula. B. Plántula con raicillas. Fotografías: John Bernal.

A. Pico), y que también se ocasiona por el consumo de semillas por parte de insectos en páramos húmedos como los de Chingaza y Sumapaz (observaciones personales de A. Pico, JBB, en P. santosii y Puya goudoutiana).

El tiempo de germinación de $P$. loca presenta un comportamiento similar a otras especies de Puya, pues se ha reportado la germinación ocurre en su mayoría antes de los 30 días después de la siembra, tal como se refiere en la Tabla 1.

Crecimiento y desarrollo. Las plántulas desarrollaron una hoja entre los días 19 y 29 después de la siembra y presentaron una altura inferior a $10 \mathrm{~mm}$; en el día 35 comenzaron a aparecer dos hojas en tres plántulas. En el día 61, 21 plántulas desarrollaron dos hojas y otras nueve, tres hojas (Tabla 2).

El comportamiento de las plántulas después de la resiembra en cuanto a su crecimiento y desarrollo a los 9,5 y 15 meses, se presenta en las Figuras 5, 6A, 6B y Tabla 2. Con 15 meses de edad, las plántulas sembradas en $6 \mathrm{~cm}$ de turba desarrollaron cinco a siete hojas y alcanzaron 6 a $7 \mathrm{~cm}$ de diámetro, y las plántulas sembradas en 2,5 a $3 \mathrm{~cm}$ de turba tenían cuatro a seis hojas y alcanzaron 3 a $4 \mathrm{~cm}$ de diámetro,
Tabla 2. Resultados de germinación, crecimiento y desarrollo ex situ de Puya loca

\begin{tabular}{|c|c|c|}
\hline $\begin{array}{l}\text { Días después } \\
\text { de la siembra }\end{array}$ & $\begin{array}{c}\text { Número de } \\
\text { plántulas (/40) }\end{array}$ & $\begin{array}{l}\text { Número de hojas, diámetro } \\
\text { y otras observaciones }\end{array}$ \\
\hline Día 19 & $\begin{array}{l}\text { Inicio de la } \\
\text { germinación } \\
8\end{array}$ & Una hoja \\
\hline Día 21 & 17 & Una hoja \\
\hline Día 29 a 61 & 30 & Una hoja \\
\hline Día 35 & 30 & $\begin{array}{l}27 \text { plántulas con una hoja } \\
\text { Tres plántulas con dos hojas }\end{array}$ \\
\hline $\begin{array}{l}\text { Día } 61 \\
(2 \text { meses })\end{array}$ & 30 & $\begin{array}{l}21 \text { plántulas con dos hojas } \\
\text { Nueve plántulas con tres hojas }\end{array}$ \\
\hline $\begin{array}{l}\text { Día } 287 \\
(9,5 \text { meses })\end{array}$ & - & $\begin{array}{l}\text { Todas con roseta conformada } \\
\text { Sustrato de } 2,5 \text { a } 3 \mathrm{~cm} \text { : plántulas } \\
\text { con tres a seis hojas verdaderas y } \\
1 \text { a } 4 \mathrm{~cm} \text { de diámetro } \\
\text { Sustrato de } 6 \mathrm{~cm} \text { : plántulas con } \\
\text { cuatro a siete hojas verdaderas y } \\
3 \text { a } 4 \mathrm{~cm} \text { de diámetro }\end{array}$ \\
\hline $\begin{array}{l}\text { Día } 448 \\
(15 \text { meses })\end{array}$ & - & $\begin{array}{l}\text { Todas con canal central foliar que } \\
\text { conduce parcialmente agua al centro } \\
\text { Sustrato de } 2,5 \text { a } 3 \mathrm{~cm} \text { : plántulas } \\
\text { con cuatro a cinco hojas verdaderas } \\
\text { y } 3 \text { a } 4 \mathrm{~cm} \text { de diámetro } \\
\text { Sustrato de } 6 \mathrm{~cm} \text { : plántulas con cinco } \\
\text { a siete hojas verdaderas (más anchas) } \\
\text { y } 6 \text { a } 7 \mathrm{~cm} \text { de diámetro }\end{array}$ \\
\hline
\end{tabular}

Nota: en el Jardín Botánico de Bogotá, Colombia, 2.550 msnm. Semillas almacenadas en condiciones ambientales durante 14 meses



Figura 5. Plántulas en bandeja de germinación con 2,5 a $3 \mathrm{~cm}$ de profundidad de turba a los 9,5 meses de la siembra. Promedio de tres a seis hojas verdaderas y 1 a $4 \mathrm{~cm}$ de diámetro, 2015. 

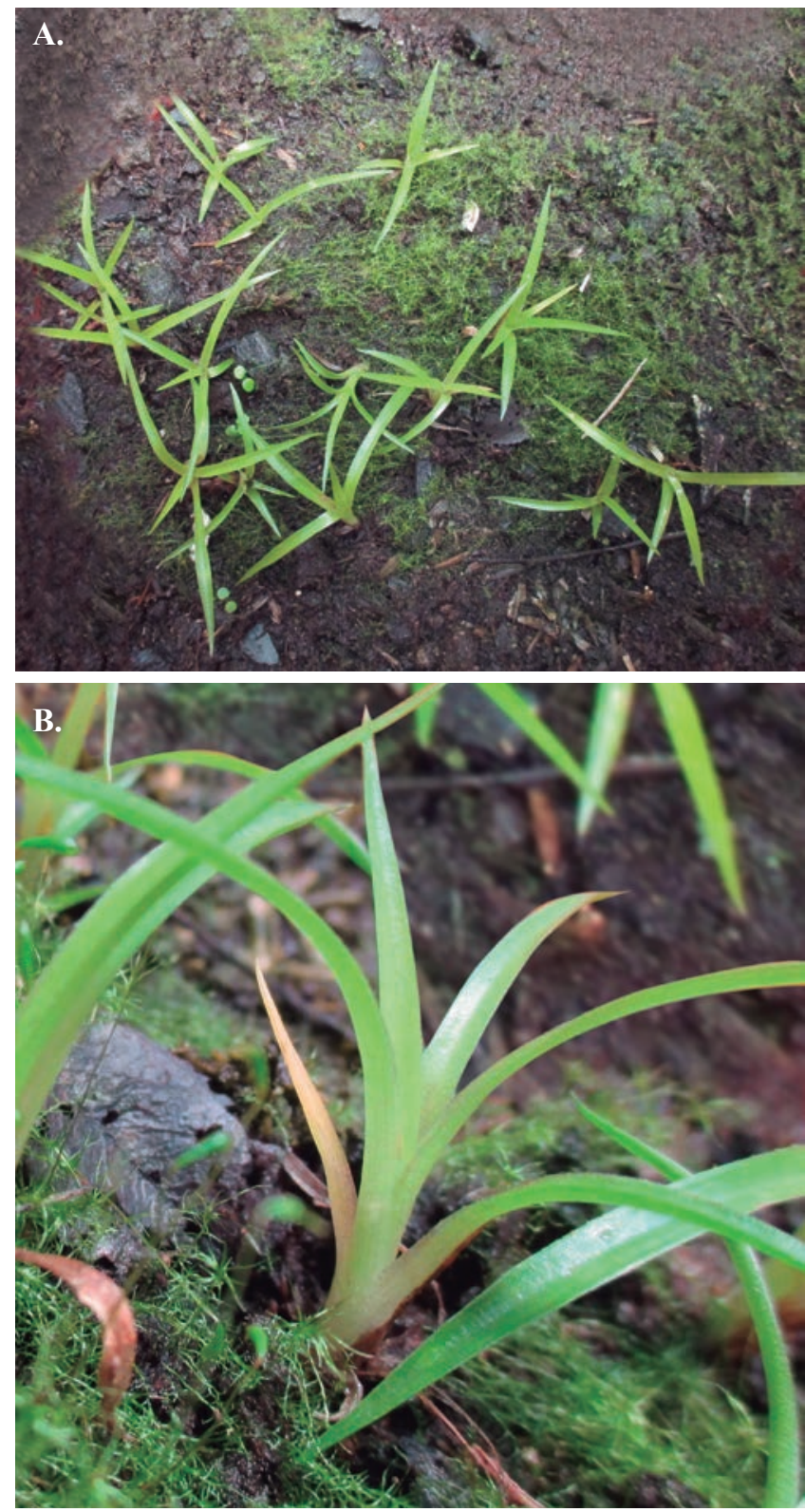

Figura 6. Se observan las plántulas resembradas en materas con 6 $\mathrm{cm}$ de profundidad de turba a los 15 meses de la siembra con un promedio de cinco a siete hojas verdaderas y 6 a $7 \mathrm{~cm}$ de diámetro, 2016. A. Grupo. B. Detalle

aunque crecieron con menor vigor. En consecuencia, se obtuvo un mayor desarrollo de las plántulas de $P$. loca en una profundidad de siembra de $6 \mathrm{~cm}$ de turba.

Se concluyó que las semillas de $P$. loca almacenadas durante 14 meses pudieron generar una población abundante de plántulas, pues $75 \%$ germinó, valor considerado entre medio y alto con relación al tiempo transcurrido desde su liberación en campo. De ahí la necesidad de sembrar las plántulas en germinadores más profundos desde el inicio de la siembra o resembrarlas antes de los nueve meses de germinadas en contenedores con mínimo $5 \mathrm{~cm}$ de profundidad.

\section{Agradecimientos}

El autor agradece al JBB por los aportes económicos y los materiales para el desarrollo de la colección viva de bromelias entre el 2013 y el 2016, especialmente a Vilma Jaimes y Mauricio Diazgranados; a Ariel López, por impulsar las exploraciones botánicas del JBB en Usme y Chipaque, y a Johanna Romero, Fabio Ávila y Germán Barrera, por la revisión del manuscrito, así como a los evaluadores anónimos por sus comentarios.

\section{Conflicto de intereses}

Declaro que no existe conflicto de intereses con respecto a ninguno de los aspectos abordados en este trabajo.

\section{Referencias}

Alcaldía Mayor de Bogotá. (2016). Acuerdo Local Número 001 (26 septiembre de 2016), por el cual se adopta el plan de desarrollo económico, social, ambiental y de obras públicas para la localidad de Usme 2017-2020. Bogotá Mejor para Todos, Usme Mejor para Todos: prospera, segura, ordenada, educada y con cultura ciudadana 2017 - 2020. Fecha de consulta: 28 de octubre de 2016. Disponible en: http://www.usme.gov.co/index.php/transparencia/planeaciongestion--control/2013-09-23-23-42-29/category/146-plan-dedesarrollo-local-2017-2020.

Betancur, J. C. (2016). Bromeliaceae. En R. Bernal, S. R. Gradstein y M. Celis (editores). Catálogo de plantas y líquenes de Colombia. p. 943-990. Bogotá: Instituto de Ciencias Naturales, Universidad Nacional de Colombia. Fecha de consulta: 29 de mayo de 2016. Disponible en: http://catalogoplantasdecolombia.unal.edu.co.

Betancur, J. \& García, N. (2006). Las bromelias. En N. García y G. Galeano (editores), Libro Rojo de las Plantas de Colombia. Volumen 3: Las Bromelias, Las Labiadas y Las Pasifloras. p. 51-384. Bogotá: Instituto Alexander Von Humboldt e Instituto de Ciencias Naturales de la Universidad Nacional.

Guhl-N., A. (2015). Transformaciones del paisaje en los páramos circundantes de la Sabana de Bogotá. En: Guhl-N., A., (editor). Los páramos circundantes de la Sabana de Bogotá. Edición conmemorativa. p. 208-231. Bogotá: Jardín Botánico José Celestino Mutis, $2^{\mathrm{a}}$ ed. Colección Pérez Arbeláez, No.5.

Jardín Botánico de Bogotá. (2015). Objetivos estratégicos y estrategias del JBB. Fecha de consulta: 13 de febrero de 2015. Disponible en: http://www.jbb.gov.co/jardin/nuestrojardin-inicio/objetivos-estrategicos.

Ministerio de Ambiente y Desarrollo Sostenible. (2014). Resolución 0192 de 2014, por la cual se establece el listado de las especies silvestres amenazadas de la diversidad biológica colombiana que se encuentran en el territorio nacional, y se dictan otras disposiciones. Bogotá. Fecha de consulta: 2 de mayo de 2016. Disponible en: http://www.icbf.gov.co/cargues/ avance/docs/resolucion_minambienteds_0192_2014.htm.

Madriñán, S. (2015). Una nueva especie de Puya (Bromeliaceae) de los páramos cercanos a Bogotá, Colombia. Rev. Acad. Colomb. Cienc. Ex. Fis. Nat. 39 (152): 389 - 398.

Mora, F., Chaparro A., Vargas, O, Bonilla, A. (2007). Dinámica de la germinación, latencia de semillas y reclutamiento de plántulas en Puya cryptantha y P. trianae, dos rosetas gigantes de los páramos colombianos. Revista Ecotropicos. 20 (1): 31-40. 
Pico-V., A. (2002). Informe final, Programa flora amenazada, Familia Bromeliaceae. Jardín Botánico José Celestino Mutis, No. 108-2002. Manuscrito no publicado.

Pico-V., A. (2014a). Germinación, crecimiento y desarrollo de Puya bicolor Mez (BROMELIACEAE). En: Informe final del contrato con el Jardín Botánico José Celestino Mutis No. 586-2013. Manuscrito no publicado.

Pico-V., A. (2014b). Germinación, crecimiento y desarrollo de Puya santosii Cuatrec. (BROMELIACEAE). En: Informe final del contrato con el Jardín Botánico José Celestino Mutis No. 586-2013. Manuscrito no publicado.

Pico-V., A. (2014c). Colecta, montaje y accesiones de Puya sp. nov. (BROMELIACEAE). En: Informe final del contrato con el Jardín Botánico José Celestino Mutis No. 586-2013. Manuscrito no publicado.

Pico-V., A. (2015). Notas acerca de hábitat, fenología y germinación de Vriesea sp. nov. y Puya sp. nov. (BROMELIACEAE), Colombia. Trabajo presentado en el VIII Congreso
Colombiano de Botánica. Universidad de Caldas - FAUC Herbario Universidad de Caldas - Asociación Colombiana de Botánica, Manizales.

Secretaría Distrital de Ambiente. 2007. Atlas ambiental de Bogotá, D.C. Imprenta Nacional de Colombia. 272 p.

The International Plant Names Index. (2016). Puya loca Madriñan. Fecha de consulta: 12 de marzo de 2016. Disponible en: http://www.ipni.org/ipni/idPlantNameSearch. do?id=60469999-2\&back page $=\% 2$ Fipni $\% 2$ FeditSimpleP lantNameSearch.do\%3Ffind_wholeName\%3Dpuya\%2Blo ca\%26output format\%3Dnormal.

The Plant List. (2013). The Plant List, género Puya. Fecha de consulta: 14 de marzo de 2016. Disponible en: http://www. theplantlist.org/.

Vadillo, G., Suni, M., Cano, A. (2004). Viabilidad y germinación de semillas de Puya raimondii Harms (Bromeliaceae). Rev. Perú. Biol. 11 (1): 71-78. 